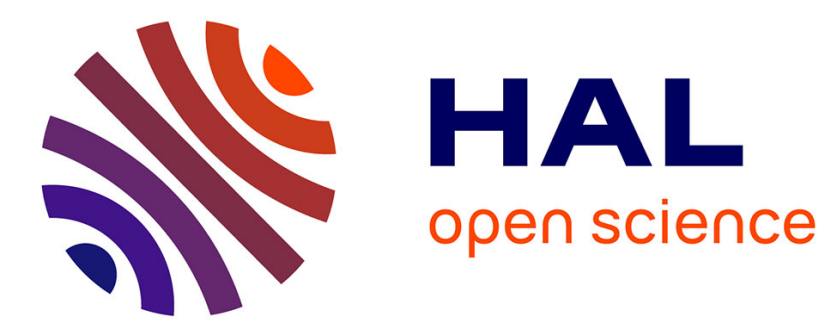

\title{
Intraoperative Biomechanical Registration of the Liver: Does the Heterogeneity of the Liver Matter?
}

Rosalie Plantefève, Igor Peterlik, Stéphane Cotin

\section{To cite this version:}

Rosalie Plantefève, Igor Peterlik, Stéphane Cotin. Intraoperative Biomechanical Registration of the Liver: Does the Heterogeneity of the Liver Matter?. Innovation and Research in BioMedical engineering, 2018, 39 (1), pp.18-25. 10.1016/j.irbm.2017.10.005 . hal-01625573

\section{HAL Id: hal-01625573 \\ https://inria.hal.science/hal-01625573}

Submitted on 27 Oct 2017

HAL is a multi-disciplinary open access archive for the deposit and dissemination of scientific research documents, whether they are published or not. The documents may come from teaching and research institutions in France or abroad, or from public or private research centers.
L'archive ouverte pluridisciplinaire HAL, est destinée au dépôt et à la diffusion de documents scientifiques de niveau recherche, publiés ou non, émanant des établissements d'enseignement et de recherche français ou étrangers, des laboratoires publics ou privés. 


\title{
Intraoperative biomechanical registration of the liver: does the heterogeneity of the liver matter?
}

\author{
Plantefève Rosalie ${ }^{\mathrm{a}, \mathrm{b}, *}$, Igor Peterlik $^{\mathrm{c}}$, Stéphane Cotin $^{\mathrm{c}}$ \\ ${ }^{a}$ LCTI, CRCHUM, Montréal \\ ${ }^{b}$ MEDICAL, Polytechnique Montréal, Montréal \\ ${ }^{c}$ MIMESIS, Inria, Strasbourg
}

\begin{abstract}
Background:

Preoperative images such as computed tomography scans or magnetic resonance imaging contain lots of valuable information that are not easily available for surgeons during an operation. To help the clinicians better target the structures of interest during an intervention, many registration methods that align preoperative images onto the intraoperative view of the organs have been developed. For important organ deformation, biomechanically-based registration has proven to be a method of choice.

Method:

Using an existing biomechanically-based registration algorithm for laparoscopic liver surgery we investigate in this paper the influence of the heterogeneity of the liver on the registration result.

Results:
\end{abstract}

Conclusion:

Keywords: Intraoperative registration, Hepatic surgery, Laparoscopic surgery, Biomechanical model

\footnotetext{
* Corresponding author

Email address: rosalie@plantefeve.fr (Plantefève Rosalie)
} 


\section{Introduction}

(a)

(c) (b)

(d)

Figure 1: (a) Laparoscopic view of the liver during a surgical intervention. (b) Computed tomography images of the pre operative liver and the segmentated mesh of the liver with its internal structures. Laparoscopic view (c) and segmented mesh (d) of the liver overlayed with two anatomical landmarks: the umbilical notch in yellow and the anterior margin in red.

Liver cancer is one of the most lethal liver pathologies with 47,000 deaths in Europe and 745,000 deaths worldwide per year. Today, surgical resection remains the best treatment for liver cancer and metastasis [1]. During this 5 intervention, the tumors are removed from the organ while ensuring that a sufficient remnant volume is preserved and well vascularized to allow for complete organ regeneration. Therefore, localizing precisely during surgery the tumors and the blood vessels is the keypoint for the success of hepatic tumor resection. As these structures are visible on preoperative medical images, image guided surgery (IGS) systems that register preoperative data onto the intraoperative view have been proposed [2, 3, 4, 5].

Among the different approaches, biomechanically based registration is the most promising. Indeed, this type of method is able to handle large organ deformation even with a limited amount of intraoperative data [6. Intraoperative information is particularly limited during laparoscopic liver tumor resection (see Fig. 11, a kind of minimally invasive surgery where the abdomen of the patient is insufflated with carbon dioxide and surgeons operate with long and thin instruments which enter through trocars placed in small apertures made in the abdominal wall. During this intervention, the liver is strongly deformed due to 2o the increase in the intra-abdominal pressure.

In [7 an IGS system relying on biomechanically-based registration for laparoscopic liver tumor resection has been proposed. In this work, the authors 
use a heterogeneous patient-specific liver model as input for their registration method. However, they did not investigate the impact of using a heterogeneous model instead of a simpler homogeneous model.

In [8] Wittek et al. reported that despite the fact that the brain is a hyperelastic material the use of an hyperelastic constitutive law for the registration of preoperative data onto the intraoperative view does not improve the results compared to the use of an elastic constitutive law.

In this article, we investigate the impact of the heterogeneity of the biomechanical liver model on the registration results in the framework presented in 7.

\section{Material and Methods}

\subsection{Registration algorithm overview}

Figure 2: Main steps of the registration method. First, a matching is performed between the selected anatomical features as well as for the whole surface. This matching is used to compute penalty forces which will deform the biomechanical model of the liver. Then, the matching is recomputed and new forces are applied on the model. The penalty forces are increased each time the registration has reached an equilibrium. This process is iterated until the convergence criterion is met.

35

The registration method used is similar to the one described in [9] and recasts the registration problem as a system of biomechanical equations. It takes as input a three-dimensional point cloud reconstructed from a stereoscopic camera view of the organ surface (see Fig. $3(\mathrm{a})$ and a biomechanical model of the liver (see Fig. 3(b) and 3(c) . Both data sets are labeled with two landmarks

40 corresponding to the umbilical notch (UN) and the anterior margin (AM) (see Fig. 11.

At the beginning of the registration process, the biomechanical model (source) is in its pre-operative configuration and needs to be deformed to match the intra-operative point cloud (target). This match is only partial since the target 
45 represents only $40 \%$ of the total surface of the liver in the best case. The biomechanical model — described in Section 2.3 — is used to simulate the behavior of the organ while the registration constraints imposed to the deformable object are modeled with penalty forces $f_{\text {ext }}$ computed from the set of correspondences defined in Section 2.2, During the registration process, theses penalty forces

so are increased progressively in an annealing scheme each time the registration process reaches an equilibrium until the convergence criterion is met. Fig. 2 summarizes the pipeline.

\subsection{Matching}

The correspondences are defined for each landmark (UN and AM, see Fig. 1) 55 and for the rest of the liver surface independently, that is, it does not allow for inter-region correspondences. For each area, the points of the target point cloud are projected onto their corresponding area on the liver surface - the source as shown in Fig. 2. We do not project the source model points on the target since the target only corresponds to a part of the source model, thus an important

60 number of matches would be inaccurate. Each target point is projected onto all the triangles of the source model surface with the same label. A matching pair $\left\{p_{t}, p_{s}\right\}$ consisting of the target point and its closest projection is then defined.

The outliers are pruned with a relative distance threshold and optionally, if the point cloud is smooth enough to accurately compute the normal at each

${ }_{65}$ point, a relative normal threshold. The relative distance threshold keeps the pairs of points for which: $\left|\mathbf{p}_{t s}\right|<d_{t} \max \left(\left\{\left|\mathbf{p}_{t s}\right|\right\}_{\text {all pairs }}\right)$, with $d_{t} \in[0 ; 1]$ where $\mathbf{p}_{t s}$ is the vector which goes from $p_{t}$ to $p_{s}$ and $|\cdot|$ is the Euclidean distance. The normal threshold prunes the pairs whose dot product is smaller than a threshold: $\mathbf{n}_{p_{t}} \cdot \mathbf{n}_{p_{s}}<n_{t}$, with $n_{t} \in[0 ; 1]$. These thresholds must be set according to the deformation characteristics and they should be smaller for larger deformations. On average $d_{t}=0.9$ and $n_{t}=0.7$.

The registration algorithm aims at minimizing the distance between the matching pairs. 
(a) Point cloud generated (b) Homogeneous model. (c) Heterogeneous model.

from the laparoscopic view.

Figure 3: Inputs of the registration method for the source and target data.

\subsection{The biomechanical models}

75 registration problem. Two biomechanical models are used, the first one being the complete heterogeneous liver model and the other one being a homogeneous model composed only of the liver parenchyma (see Fig. 3).

The heterogeneous liver model (see Fig. 3(c) is defined as in [7] using the finite element formulation and is composed of three parts: the parenchyma modeled as co-rotational tetrahedral elements, the Glisson's capsule modeled as a membrane using constant strain triangular elements, and the vascular tree modeled as Tymoshenko beam elements Their hollow structure is taken into account via the proper definition of their moments of inertia. The homogeneous

85 liver model is composed only of the parenchyma.

\subsection{External forces}

For each pair $\left\{p_{t_{i}}, p_{s_{i}}\right\}$ we define an external force:

$$
\mathbf{f}_{e x t_{i}}=k\left(1-\frac{2}{\pi} \arctan \left(\sigma\left|\mathbf{p}_{s t_{i}}\right|\right)\right) \frac{\mathbf{p}_{s t_{i}}}{\left|\mathbf{p}_{s t_{i}}\right|}
$$

where $k$ is a scalar stiffness coefficient (in $[\mathrm{N} / \mathrm{m}])$ and the term $\left(1-\frac{2}{\pi} \arctan \left(\sigma\left|\mathbf{p}_{s t_{i}}\right|\right)\right.$ is an asymptotic penalty function of the distance (in [m]) which includes also the scale factor $\sigma$. The distance |.| is the Euclidean distance. This scale factor is used to avoid over-fitting when the pre-operative model is registered to a noisy point cloud: a lower value of $\sigma$ decreases the force intensity for small distances. In practice, we set: $\sigma=\tan \left(0.9 \frac{\pi}{2}\right) \frac{1}{d_{90}}$ where $d_{90}$ is the distance for which the force magnitude is equal to $90 \%$ of its maximum.

As in [9] we define a different stiffness coefficient for each feature $k_{U N}, k_{A M}$ and $k_{\text {surf }}$ where the identifiers stand for the umbilical notch, the anterior margin 
and the remaining surface, respectively and we let them evolve during the reg-

istration process: $k_{j_{\min }}+\frac{n^{e}}{r_{j}^{e}}\left(k_{j_{\max }}-k_{j_{\min }}\right)$ for $n<r_{j}$ and $k_{j_{\max }}$ for $n \geq r_{j}$ where $j \in\{U N, A M, \operatorname{surf}\}, n \geq 0$ is the plateau index which is incremented each time the registration process has reach is optimum for the actual stiffness coefficients, $r_{j}>0$ an integer which controls the increase rate of $k_{j}$ and $e$ an exponent which control the shape of the force intensity evolution. Experimentally, we found that setting $e=3$ leads to a better robustness of the algorithm. The convergence criterion of this registration process is not defined by a threshold on the residual error. Indeed, this error can only be computed on the visible surface and depends highly on the data noise. Thus, the registration is stopped when the maximal stiffness is reached for all features, that is as soon as $n=\max \left(r_{j}\right)+1$.

\subsection{Registration methods}

As our registration problem has been recast into a biomechanical problem, we solve it using the solvers developed for this type of system. Similarly as in [6, we consider the registration as a dynamic process. This avoids having to set Dirichlet boundary conditions such that the stiffness matrix is invertible. Such boundary conditions would not make sense as the initial position of the biomechanical model may be far from the target point cloud at the beginning of the registration. The dynamic system of equations is given by: $\mathbf{M} \ddot{\mathfrak{u}}=\mathbf{K} \mathfrak{u}+$ $\mathbf{D} \dot{\mathfrak{u}}+\mathbf{f}_{\text {ext }}$ where $\mathbf{M}$ is the mass matrix, $\mathbf{D}$ is the damping matrix, $\mathbf{K}$ is the stiffness matrix, and $\mathfrak{u}$ is the vector of nodal displacements. At each step of the registration process, this dynamic system is integrated in time using implicit backward Euler scheme.

After each resolution of the system, the pairing is recomputed, generating a new force. Thus, the system does not reach its equilibrium position for a certain matching before it is updated. This helps the matching to converge toward its optimum in case of large initial misalignment. Finally, during the simulation, the anatomical landmarks provide a coarse registration that improves the robustness 125 of the matching algorithm, whereas the biomechanical model plays a role of 
regularization and allows for an accurate solution of local deformations.

\subsection{Evaluation of the registration method}

The aim of the method is to estimate the location of internal structures of the organ which undergoes important intraoperative deformations when compared to its initial pre-operative configuration. Validating the registration in this context is very challenging since the optimal ground truth would be a $3 \mathrm{D}$ reconstruction of the organ at the intra-operative stage, which requires the intraoperative CT or MR scan of the patient, and a mean to track the position of the laparoscope relatively to the patient anatomy. To the best of our knowledge,

135 such data does not exist. Indeed, access to these techniques is very limited and almost impossible to use on human subjects. On the other hand, using swines to validate the registration method would be an issue since the geometry and the surrounding environment of a swine liver differ significantly from a human liver and thus the deformation induced by the pneumoperitoneum is very different in 140 the two cases. Therefore, we used synthetic data to validate quantitatively our registration method. This offers the advantage to control all the parameters, thus allowing for a better characterization of the method.

\subsubsection{Generation of synthetic data}

In order to obtain the ground truth for the registration, we have deformed the biomechanical models using a physical simulation of a pneumoperitoneum. As we control the mechanical properties of the biomechanical model used for the simulation we can measure the influence of the biomechanical model used for the registration. Two sets of parameters were used; one representing a healthy liver (parameter values are set as reported in [10]) where $E$ is the Young's modulus and $\nu$ is the Poisson's ratio:

$$
\begin{array}{ll}
E_{\text {parenchyma }}=3.5 \mathrm{kPa}, & \nu_{\text {parenchyma }}=0.45, \\
E_{\text {vessels }}=620 \mathrm{kPa}, & \nu_{\text {vessels }}=0.4, \\
E_{\text {capsule }}=8000 \mathrm{kPa}, & \nu_{\text {capsule }}=0.45,
\end{array}
$$


and the other representing a cirrhotic liver with $E_{\text {parenchyma }}=30 \mathrm{kPa}$, all the other parameters are identical to the one of the normal liver.

Figure 4: Non-deformed and deformed configurations of deformation \#3 and \#4. The top row shows a view of the anterior liver surface and The first and the bottom row a view of the posterior and inferior liver surface. third columns show the non-deformed configuration, the second column the deformed configuration obtained using the cirrhotic mechanical parameters and the fourth column the deformed configuration obtained using the healthy mechanical parameters. The colors correspond to the relative Hausdorff distance between the deformed and the non-deformed configurations for the cirrhotic and healthy liver.

In total, we use four deformations mimicking the pneumoperitoneum generated using the different mechanical parameters given above. All deformations were obtained using a heterogeneous liver model. Deformations \#1 and \#3 were obtained using the cirrhotic mechanical parameters while deformations \#2 and

150 \#4 were obtained using the healthy mechanical parameters. Deformations \#3 and $\# 4$ are shown in Fig. 4 .

Figure 5: Partial surface generated from deformation \#3. Top row corresponds to front view and bottom row to back view. The first column corresponds to $50 \%$ of the entire surface, the second to $40 \%$, the third to $30 \%$, the fourth to $20 \%$ and the fifth to $10 \%$. The data are represented as surfaces but the algorithm ignore the topology and the normals of these surfaces.

In real situations, only partial surface information is acquired by the laparoscopic camera. Thus, to evaluate the amount of information needed to achieve an accurate registration faces of the deformed model are deleted to keep only a portion of its surface from $50 \%$ to $10 \%$ (see Fig. 5 ).

\subsubsection{Error measure}

To measure the accuracy of the registration, several error measures are used. For synthetic data two quantitative error measures are employed: the Hausdorff distance and the target registration error (TRE) between the degrees of freedom of the finite element mesh. Mathematically, the Hausdorff distance $H_{D}$ is a measure which quantifies the distance between two surfaces $S_{1}$ and $S_{2}$, and is 
defined as follows:

$$
H_{D}=\max \left(\max \left(\min _{s_{1} \in S_{1}} d\left(s_{1}, S_{2}\right)\right), \max \left(\min _{s_{2} \in S_{2}} d\left(s_{2}, S_{1}\right)\right)\right)
$$

where $d\left(s_{i}, S_{j}\right)$ is the Euclidean distance between the point $s_{i}$ and the surface $S_{j}$. However, in practice, the algorithm which calculates the Hausdorff distance samples a certain number of points $p$ on one surface, computes their distances to the other surface and gives statistics on the measured distances. Thus, in the following, we use the mean and the maximum Hausdorff distances, which are respectively the mean and the maximum of the sampled distances:

$$
\begin{gathered}
H_{D \text { max }}=\max \left(\max \left(\min _{p_{1} \in P_{1}} d\left(p_{1}, S_{2}\right)\right), \max \left(\min _{p_{2} \in P_{2}} d\left(p_{2}, S_{1}\right)\right)\right) \\
H_{\text {Dmean }}=\operatorname{mean}\left(\min _{p_{1} \in P_{1}} d\left(p_{1}, S_{2}\right), \min _{p_{2} \in P_{2}} d\left(p_{2}, S_{1}\right)\right)
\end{gathered}
$$

where $P_{1}$ and $P_{2}$ are the sets of sampled points on surface $S_{1}$ and $S_{2}$ respectively. The TRE is the distance between two points which correspond to the same position on the source and on the target and that have not been used in the registration algorithm.

\section{Results}

Table 1: Statistics on the deformations obtained with heterogeneous models. Deformations \#1 and \#2 are small compared to deformations \#3 and \#4.

To evaluate the registration method, the undeformed healthy and cirrhotic biomechanical models were registered onto the partial surfaces generated in Section 2.6.1. Then, the shapes of the models after registration were compared with the deformed configurations of the whole meshes used for the generation of partial surfaces. For all registrations we adjusted the stiffness parameters $k_{j}$ for $j \in\{U N, A M$, surf $\}$ according to the Young's modulus. In this manner, we ensured that the ratio between the external and the internal forces remains the same independently of the actual value of the Young's modulus. We use 170 a conversion factor $10^{-3} \mathrm{~m}$ for $k_{U N_{\max }}, k_{A M_{\max }}$, and $k_{\text {surf }}$ max , a factor $10^{-4} \mathrm{~m}$ 
for $k_{U N_{\min }}$ and we set $k_{\text {surf } \min }=0 \mathrm{~N} \cdot \mathrm{m}^{-1}, r_{U N}=2, r_{A M}=4$, and $r_{\text {surf }}=10$. For the implicit Euler integration scheme, the Rayleigh mass and the Rayleigh damping coefficients were set to 0.1 . For all tested scenarios, the computational time of the method is under 2 minutes, which is acceptable for an application in 175 the operating room. In order to ensure the success of the method, the anatomical features must be at least partially visible.

Figure 6: Distances between the degrees of freedom of the finite element surface and volume mesh between different deformed configurations. A) heterogeneous liver model: cirrhotic (deformation \#1) vs. healthy (deformation \#2), B) healthy liver: homogeneous vs. heterogeneous model, C) cirrhotic liver: homogeneous vs. heterogeneous model.

Statistics on the deformations are given in Table 1 and Fig. 6. The figure shows that the presence of the vessels and the capsule influences the deformation, particularly in the case of the healthy liver where the mean distance between the degrees of freedom of the final configuration of the heterogeneous and homogeneous model is $10.1 \mathrm{~mm}$. This error represents $43 \%$ of the homogeneous healthy liver deformation. The same analysis performed for the cirrhotic liver quantifies the influence of the vessels being $18 \%$ w.r.t. the entire deformation.

\subsection{Influence of the mechanical parameters}

We tested the impact of the relative stiffness of these three components of the liver on the registration accuracy. The results are presented in Fig. 7 . The maximum target registration error (TRE) obtained by the registration of the healthy liver on the cirrhotic deformation (deformation \#4) are slightly better than the maximum TRE obtained by the cirrhotic liver. Nonetheless, if we compare the mean TRE and the values of the first and third quartile, the results do not show a statistically significant difference between the use of an healthy or a cirrhotic liver to perform the registration, indicating that the exact value of the parenchyma Young's modulus is not necessary to obtain accurate results. 
Figure 7: Comparison of the TRE obtained using the cirrhotic or healthy liver for the registration task on deformation \#3 (a) and using the cirrhotic or healthy liver for the registration task on deformation \#4 (b).
(a) Cirrhotic liver. Deformation \#1
(b) Healthy liver. Deformation \#2
(c) Cirrhotic liver. Deformation \#3
(d) Healthy liver. Deformation \#4

Figure 8: Comparison of the TRE obtained after registration with the homogeneous and heterogeneous liver model using deformation \#1, \#2, \#3 and \#4.

\subsection{Homogeneous versus heterogeneous biomechanical models}

Additionally, we studied the impact of using a simplified homogeneous model on the registration results. We compared the registration accuracy obtained for the complete heterogeneous liver model composed of the Glisson's capsule, the vascular tree and the parenchyma with the results obtained using a simplified model composed only of the liver parenchyma. The results are presented in Fig. 8 and show that using a complete liver model does not significantly improve the registration accuracy. Moreover, the use of a heterogeneous liver model increases the computation time by a factor of two compared to the same registration task performed with a homogeneous model.

\section{Discussion}

Our results show that the biomechanically-based registration method is able to perform well even with challenging registration problem where the amount of visible surface is very limited. Moreover, the fast computational time makes this method well suited for the alignment of pre- and intra-operative data for laparoscopic liver surgery.

As expected the results show that using different mechanical model and mechanical properties to generate deformations leads to significant differences 
between the deformed states (see Fig. 6). However, setting the mechanical properties of the registered biomechanical model to those used to obtain the de-

215 formed state does not necessarily lead to better results than when using slightly different values (see Fig. 7).

The results also suggest that the heterogeneous liver model does not improve significantly the registration result. Indeed, excluding the results obtained for $10 \%$ of visible surface, the difference in accuracy between the two models is on average of $0.29 \mathrm{~mm}$, which represents a relative error of $2.5 \%$ with respect to the deformations, and the homogeneous model performs sometimes better. Moreover, as the computational time is increased when using a heterogeneous model, the homogeneous biomechanical model should be preferred for this specific application. This ties to the results reported by Wittek et al. in [8] and can be

225 due to the particularities of the deformations - that mimic the pneumoperitoneum - applied to the liver or to the problem formulation. Nonetheless, the value of the mechanical parameters and external forces should be defined to avoid overfitting while ensuring that external forces are strong enough to deform the biomechanical model. Thus, the ratio between the liver stiffness and the external force intensity should be chosen carefully. In this work, we use realistic mechanical parameters for healthy and cirrhotic livers, and our choice of external forces magnitude was selected to be in the range of the force that the carbon dioxide exercise on the liver during the pneumoperitoneum which is responsible for the organs deformation.

\section{Conclusion}

In this article, we studied the impact of different mechanical models on a bio-mechanically-based preoperative to intraoperative liver registration using a point cloud reconstructed from a stereoscopic camera as target. Our results show that using a heterogeneous liver model not improve the registration results but increases the computational time. Thus we recommend using the homogeneous model in this registration framework. Moreover, the exact value of the 
mechanical parameters has only little influence on the registration result, suggesting that with the current problem formulation the knowledge of the patient's liver mechanical parameter is unnecessary. Future work should be conducted 245 to determine to what extend the mechanical parameters can be modified without impacting the registration results. But, this also raises a question: if the influence of the biomechanical model is limited is it still worth using it? The strength of the biomechanical model is that it only allows physical deformations, a feature that no other registration algorithm can ensure. The question is thus more: how to better use the biomechanical model in this problem? A solution to investigate would be to combine a patient-specific biomechanical simulation of the pneumoperitoneum with the proposed registration method.

Conflict of interest: none.

255

\section{References}

[1] G. Torzilli, J. Belghiti, N. Kokudo, T. Takayama, L. Capussotti, G. Nuzzo, J.-N. Vauthey, M. A. Choti, E. De Santibanes, M. Donadon, et al., A snapshot of the effective indications and results of surgery for hepatocellular carcinoma in tertiary referral centers: is it adherent to the easl/aasld recommendations?: an observational study of the hcc east-west study group, Annals of surgery 257 (5) (2013) 929-937.

[2] D. M. Cash, M. I. Miga, T. K. Sinha, R. L. Galloway, W. C. Chapman, Compensating for intraoperative soft-tissue deformations using incomplete surface data and finite elements, Medical Imaging, IEEE Transactions on 24 (11) (2005) 1479-1491.

[3] D. M. Cash, M. I. Miga, S. C. Glasgow, B. M. Dawant, L. W. Clements, Z. Cao, R. L. Galloway, W. C. Chapman, Concepts and preliminary data 
toward the realization of image-guided liver surgery, Journal of Gastrointestinal Surgery 11 (7) (2007) 844-859.

[4] M. I. Miga, P. Dumpuri, A. L. Simpson, J. A. Weis, W. R. Jarnagin, The sparse data extrapolation problem: strategies for soft-tissue correction for image-guided liver surgery, in: SPIE Medical Imaging, International Society for Optics and Photonics, 2011, pp. 79640C-79640C.

${ }_{275}^{275]}$ D. C. Rucker, Y. Wu, J. E. Ondrake, T. S. Pheiffer, A. L. Simpson, M. I. Miga, Nonrigid liver registration for image-guided surgery using partial surface data: A novel iterative approach, in: SPIE Medical Imaging, International Society for Optics and Photonics, 2013, pp. 86710B-86710B.

[6] S. Suwelack, S. Röhl, S. Bodenstedt, D. Reichard, R. Dillmann, T. dos Santos, L. Maier-Hein, M. Wagner, J. Wünscher, H. Kenngott, et al., Physicsbased shape matching for intraoperative image guidance, Medical physics 41 (11) (2014) 111901.

[7] R. Plantefève, I. Peterlik, N. Haouchine, S. Cotin, Patient-specific biomechanical modeling for guidance during minimally-invasive hepatic surgery, Annals of biomedical engineering 44 (1) (2016) 139-153.

[8] A. Wittek, T. Hawkins, K. Miller, On the unimportance of constitutive models in computing brain deformation for image-guided surgery, Biomechanics and modeling in mechanobiology 8 (1) (2009) 77-84.

[9] R. Plantefève, N. Haouchine, J.-P. Radoux, S. Cotin, Automatic alignment of pre and intraoperative data using anatomical landmarks for augmented laparoscopic liver surgery, in: Biomedical Simulation, Springer, 2014, pp. $58-66$.

[10] S. Umale, S. Chatelin, N. Bourdet, C. Deck, M. Diana, P. Dhumane, L. Soler, J. Marescaux, R. Willinger, Experimental in vitro mechanical characterization of porcine Glisson's capsule and hepatic veins., Journal of biomechanics 44 (9) (2011) 1678-83. 